optic axis and $100 \mu$ thick. From the birefringence data, values of the retardation (that is, birefringence multiplied by thickness) can be readily obtained in each case. Let these be $r_{1}$ and $r_{2}$ respectively. When the plates are crossed, the resulting retardation is $\left(r_{1}-r_{2}\right)$, and this is found to be about 6 per cent below three-quarter wavelength for most of the wavelengths considered. By increasing the thickness of the magnesium fluoride plate to $101 \mu$, its retardation becomes $\left(n_{e}-n_{o}\right) \times 101 \mu=r_{3}$, and that of the combination is then $\left(r_{3}-r_{2}\right)$ which is nearly equal to three-quarter wavelength from 303.4 to $253.7 \mathrm{~m} \mu$. Finally, values of the phase difference $(P . D)=.360^{\circ}$ $\left(r_{3}-r_{2}\right) / \lambda$ were calculated, and are shown in the last column of Table 1 .

\section{Stotherd Mitchel}

Rosebank,

Bishopbriggs.

${ }^{1}$ Mitchell, S., Nature, 166, 434 (1950); J. Sci. Instrum., 34, 89 (1957).

${ }^{3}$ Gaudefroy, M. C., C. R. Acad. Sci., Paris, 189, 1289 (1929).

${ }^{3}$ West, C. D., and Makas, A. S., J. Opt. Soc. Amer., 39, 791 (1949).

' Bruhat, G., and Weil, L., Revue d'Optique, 15, 94 (1936).

- See Landolt-Börnstein Tables, sixth ed., 8, (2), 427 (1962).

\section{Turbulent Flow Properties of Dilute Polymer Solutions}

Recentuy, Gadd ${ }^{1,2}$ and Hoyt ${ }^{3}$ reported results of experiments on the turbulent flow characteristics of dilute polymer solutions, and showed that the addition of very low concentrations of polymer molecules (of the order of 50 weight parts per million) to water greatly reduced the turbulent friction acting at surfaces moving relative to the fluid. This phenomenon was attributed to the formation of normal stress differences, because other physical propertios such as density and viscosity are sensibly unchanged. Work carried out in this department on the flow of very dilute polymer solutions and other additives in water confirms these findings. Measurements have been made of the turbulent friction factor for flow of these solutions in glass and metal pipes of varying surface roughnesses for Reynolds numbers up to 12,000 and for flow past arrays of spheres. Up to 25 per cent reduction in friction factor was observed in flow in tubes. Although we agree on the formation of normal stress differences on addition of small traces of polymeric substances to water, we would also suggest that in some instances adsorption of the solute at the solid surface can occur which will influence the flow in the boundary layer near the solid surface. Similar work carried out by El'perin and Smolskii confirms this ${ }^{4}$. The importance of these surface effects can be easily demonstrated in pipes, because it has been shown that when the flow of the solution is stopped and water reintroduced the friction factor measured is initially lower than that expected. This effect can persist for up to $15 \mathrm{~min}$, dependent on the flow conditions, and is several orders of magnitude greater than the residence time of the fluid in the tube.

At the present time we have not been able to assess quantitatively the relative importance of each mechanism.

G. A. Davies

A. B. PONTER

Department of Chemical Engineoring,

University of Manchester Institute of

Science and Technology.

${ }^{1}$ Gadd, G. E., Nature, 206, 463 (1965).

2 Gadd, G. E., Nature, 211, 169 (1966).

${ }^{3}$ Hoyt, J. W., Nature, 211, 170 (1966).

- El'perin, I. T., and Smolskii, B. M., Vesti. Akad. Navuk Belarusk־S.S.R. Sev. Fiz-Tekhn, Navuk, 2, 39 (1965).

\section{Observations of the Magnetic Dipole Rotation Spectrum of Oxygen}

Preliminary work $^{1}$ gave a spectrum covering the wavelength range $1 \mathrm{~mm}$ to $300 \mu$ of solar radiation passing through the atmosphere under conditions of low total water vapour. The expected absorption features resulting from the pure rotation lines of water vapour were recorded, but additional absorption features were present which could not be attributed to water and which in the earlier account ${ }^{1}$ were tentatively ascribed to ozone, as this is the only other major atmospheric constituent with a permanent electric dipole moment. Subsequent experimental observations on ozone in the laboratory together with the analysis of E. K. Gora ${ }^{2,3}$ showed that this explanation was not correct. About 3 years ago it was suggested that the absorption features at $14 \cdot 2,16.2$ and $27 \cdot 8 \mathrm{~cm}^{-1}$ (Fig. 1) could be attributed to molecular oxygen which has a permanent magnetic dipole moment. Transitions had been predicted which provided a plausible explanation of the observed features, but no observations of the magnetic dipole rotation spectrum had been reported. The present work was undertaken to check at least qualitatively that this absorption was as predicted; this communication reports our preliminary results.

The experimental difficulty in this research stems from the fact that magnetic dipole transitions give absorption lines which are very weak by comparison with those arising from electric dipole transitions. The ratio of these line strengths is of the order of $10^{5}$. In the solar observations the equivalent path through oxygen had been about one kilometre-atmosphere, and theoretical predictions $s^{4,5}$ indicated that a laboratory investigation would require a path length of at least $100 \mathrm{~m}$ and several atmospheres pressure. Initially, experiments were carried out in an

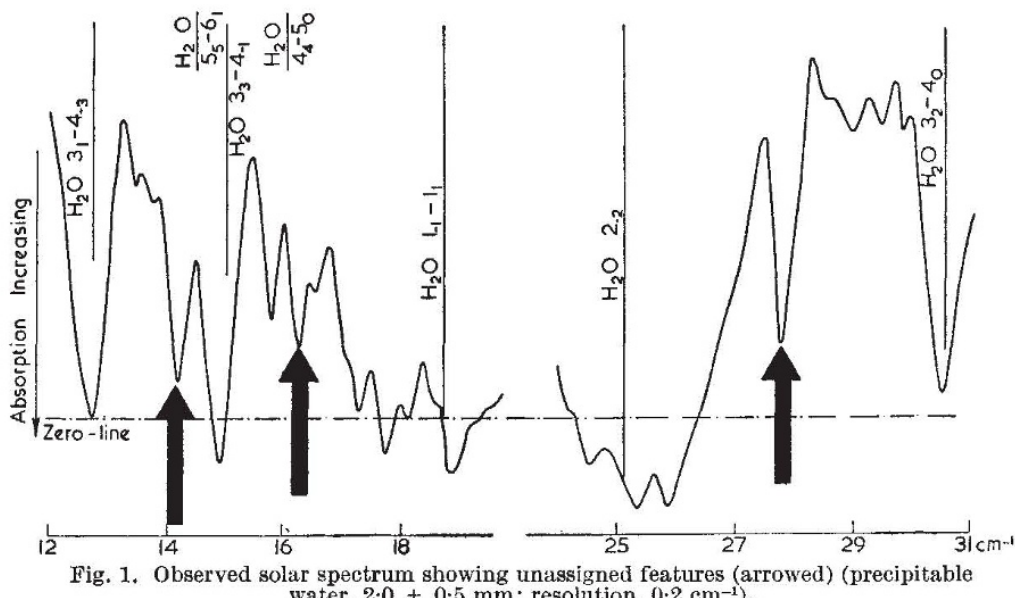

water, $2.0+0.5 \mathrm{~mm}$; resolution, $0.2 \mathrm{~cm}^{-1}$ )

absorption cell with White's optical arrangement ${ }^{5}$ having mirrors of radius of curvature $8 \mathrm{ft}$. Some results, using this cell with a path length of $110 \mathrm{~m}$ and a pressure of $6 \mathrm{~atm}$. but with a resolution limit of only $1 \mathrm{~cm}^{-1}$, were given in the National Physical Laboratory Report 1965 , and showed some expected features of the magnetic rotation spectrum. We therefore continued the research using a larger cell with mirrors of radius of curvature $24 \mathrm{ft}$.

In the second experiment we used higher spectral resolution to avoid the necessity for high pressures. Published work on the microwavo oxygen line at $4 \mathrm{~cm}^{-1}$ using microwave techniques ${ }^{6}$ had indicated that the total half width of the magnetic rotation lines would be of the order of $0.1 \mathrm{~cm}^{-1}$ per atm. Thus, by using a spectral resolution of $0.15 \mathrm{~cm}^{-1}$, a pressure of 2 atm. would be sufficient to observe the peak intensities of the absorption lines. In this way we reduced the total amount of gas 